\title{
Maximising the benefit of integrated PET/CT: the road ahead
}

\author{
von Schulthess, Gustav K
}

DOI: https://doi.org/10.1007/s00259-004-1685-8

Posted at the Zurich Open Repository and Archive, University of Zurich ZORA URL: https://doi.org/10.5167/uzh-155842

Journal Article

Published Version

Originally published at:

von Schulthess, Gustav K (2004). Maximising the benefit of integrated PET/CT: the road ahead. European Journal of Nuclear Medicine and Molecular Imaging, 31(11):1462-1463.

DOI: https://doi.org/10.1007/s00259-004-1685-8 


\title{
Maximising the benefit of integrated PET/CT: the road ahead
}

\author{
Gustav K. von Schulthess ${ }^{1}$ \\ 1 University Hospital Zurich, Zurich, Switzerland \\ Published online: 5 October 2004 \\ (C) Springer-Verlag 2004
}

Eur J Nucl Med Mol Imaging (2004) 31:1462-1463

DOI 10.1007/s00259-004-1685-8

The article by Reinartz et al. raises interesting and important issues regarding PET-CT by comparing PET and separately acquired CT. The major conclusions of the authors are twofold. First, they conclude that only a relatively small number of patients would potentially benefit from PET-CT, given that the classification of a lesion remains equivocal in only a small number of patients when PET and CT are carefully evaluated side-by-side. Second, they report that in almost half their patients, proper interpretation of a PET scan requires the availability of a recent $\mathrm{CT}$ scan for comparison. This fraction increases to two-thirds if only those PET scans that show disease are taken into account. This article is eye-opening with regard not only to the actual findings but also to the bias induced by the vantage point from which the data are considered. PET-CT is a combined modality system. The authors view PET-CT from a nuclear medicine perspective, but it may also be illustrative to consider PET-CT from a radiologist's or, better still, a future integrated modality imager's perspective.

From the vantage point of nuclear medicine, the really dramatic finding in this study is that almost half of the PET scans could not be properly interpreted alone, with CT also being required in order to properly classify the findings. When only lesion-positive PET scans were taken into account, this figure rose to $67 \%$. It would be interesting to ask what fraction of PET experts carefully analysed CT scans together with PET scans prior to the pre-PET-CT era. I freely admit that our team did not do so consistently before we started to operate our PET-CT

This Editorial Commentary refers to the article http://dx.doi.org/10.1007/s00259-004-1593-y.

Gustav K. von Schulthess (

University Hospital Zurich, Zurich, Switzerland

e-mail: vonschulthess@dmr.usz.ch

scanner in early 2001 . The data of Reinartz et al. amply demonstrate that molecular and anatomical imaging modalities are complementary, and that an intellectual synthesis of the findings of the two modalities is extremely relevant. Interpreting a PET scan without a corresponding CT scan is apparently close to unacceptable medical practice.

From a nuclear medicine point of view, there are thus significant advantages of PET-CT:

1. If it is indeed the case that half of all PET scans and two-thirds of positive PET scans need CT for proper interpretation, it would certainly be very convenient to acquire CT together with PET in a "one-stop shop" procedure. With this approach, the necessary data are always available, and they are available in a form where electronic fusion of PET and CT images poses no major problems.

2. In PET-CT, attenuation correction resulting in consistent lesion activity and allowing PET standardised uptake value measurements comes virtually free. The higher cost of the systems is offset by the higher patient throughput.

3. Fast transmission correction has the added advantage that fluorodeoxyglucose (FDG) and other ${ }^{18} \mathrm{~F}$-based radiopharmaceuticals can be used more efficiently, thereby decreasing the per-dose cost of FDG.

Thus, from a nuclear medicine perspective, PET-CT is advantageous because it is practical, even though most lesions can be classified when interpreting PET and CT side-by-side. CT, whether fused or separate, helps to specify lesions: experience of the potential value of radiology in this respect was first gained 30 years ago when comparing bone scans and X-rays of osseous and joint lesions. But, as then, the radiological part of the examination offers much more than just a gain in specificity.

From a radiologist's perspective, things look different, and the article by Reinartz et al. does not discuss this. As clinicians become accustomed to PET-CT-and they will quickly learn that PET without $\mathrm{CT}$ is less than half as good-they will no longer order a CT scan and then a separate PET scan, but will rather refer patients directly to PET-CT instead. Then the issue will be opti- 
misation not only of PET during PET-CT, but also of the CT component, so that all information relevant to a given diagnostic problem is obtained, thereby providing a true one-stop shop examination. How to perform the CT portion of the examination to provide an adequate one-stop shop in various diagnostic settings is a key question that will require substantial clinical research work over the coming years. Some examinations may be adequate with unenhanced CT only, while other diagnostic situations will require the $\mathrm{CT}$ capabilities to their maximum. Thus, the trend in the industry to provide PET-CT scanners with high- to medium-end CT scanners is understandable.

What are the additional items of information that a CT scan offers over and above that provided by a PET scan? There is much relevant diagnostic information which can be gained only with CT. In addition to the precise lesion localisation afforded by CT in PET-CT, CT but not PET is able to visualise disseminated small pulmonary metastases, pleural effusions and other fluid collections such as ascites or cystic structures, alterations in lung and liver parenchyma, and calcifications, thereby frequently offering additional relevant diagnostic information. With contrast enhancement, the relation of lesions to vascular structures can be defined with CT; such information may be of crucial relevance to the surgeon when operating on patients with central bronchial carcinoma or tumours affecting the upper abdomen and specifically the liver. All this additional information, accessible using simple or more advanced CT imaging protocols, may play a critical role in making the correct diagnosis or providing accurate staging information. CT thus contributes sensitivity in addition to specificity. Reinartz et al. in essence ignore the CT information, as they are using CT only to improve the classification of the PET lesions. They do not ask the question, "What can CT itself add to PET-CT?"

PET-CT has thus far been primarily in the hands of nuclear physicians. Various solutions have been proposed to the question of how to gain the radiological competence needed to properly interpret the CT data. Frequently, nuclear physicians interpret the PET data and radiologists the $\mathrm{CT}$ data, and then discuss the case. This may be a reasonable start, particularly when substantial cross-training occurs, but it is certainly not cost effective. It seems unfortunate that PET-CT is in danger of becoming the subject of a "turf war": radiologists are well aware of the power of PET and therefore want to "get a piece of the action", while nuclear physicians realise that PET-CT is one or perhaps the avenue to their future and want to keep PET-CT for themselves. Despite this turf war, it is clear that competence in both fields is required, and few directors of nuclear medicine or radiology services are fortunate enough to be doubly board certified. The "turf problem" will become even more relevant with the introduction of more potent SPECT-CT systems. However, while turf battles are currently being fought over power or money, they will be irrelevant in the future, because competence will prevail. This competence is to be acquired, as always, through learning. $\mathrm{Nu}-$ clear physicians in training readily learn cross-sectional imaging as they read PET-CT scans, and many young radiologists are now again opting to obtaining double board certification. They understand that molecular imaging is important and in essence resides with nuclear medicine, because even the best method in this respect to be "owned" by the radiologists, namely magnetic resonance imaging, has major limitations with regard to molecular imaging.

So what is to be done in this situation? I believe there is an urgent need for the imaging community to come together to define ways in which board-certified competence in dual-modality imaging can be obtained. Ultimately the most reasonable approach will be the formation of imaging departments which resemble neither radiology nor nuclear medicine departments but which offer board certification and subspecialisation titles in the various, relatively disparate fields of imaging and image-guided therapy. PET-CT may well be the imaging technology which allows both parties to stop feuding, to take a "fresh look at the battlefield" and to redraw "fences" in ways which make sense rather than being based on power and monetary issues only. I believe that we owe this to our young colleagues, who work hard in learning the necessary prerequisites for success in medical imaging. We should think less of defending our "turf" and more of defining reasonable prerequisites for board certification so that our residents will become respected partners of our clinical colleagues. Both nuclear medicine and radiology have developed so fast that there is still plenty of ground to cover. Only through unity will we be able to maintain the highest training standards, and this is the key to preventing clinicians from starting to perform these examinations by themselves. In oncology this may not be a major issue, but if a new generation of PET-CT or SPECT-CT scanners were able to provide a "one-stop shop" examination for the heart, this could become critical. Let us prepare now! A quote from Mikhail Gorbachev may be relevant in this context: "those who come late will be punished by life". 